\title{
Strain and dipole effects in covalent-polar semiconductor superlattices
}

\author{
Inder P. Batra* \\ IBM Research Division, Almaden Research Center K62/282, 650 Harry Road, San Jose, California 95120-6099 \\ S. Ciraci and E. Özbay ${ }^{\dagger}$ \\ Department of Physics, Bilkent University, Bilkent 06533, Ankara, Turkey
}

(Received 27 December 1990; revised manuscript received 18 April 1991)

\begin{abstract}
The energetics and electronic structure of lattice-matched $(\mathrm{Ge})_{4} /(\mathrm{GaAs})_{2}$ and strained, pseudomorphic $(\mathrm{Si})_{4} /(\mathrm{GaAs})_{2}(001)$ semiconductor superlattices have been studied with use of a self-consistent-field pseudopotential method. The interfaces are assumed to be uniform, but the interlayer distances of the pseudomorphic lattice are optimized to achieve a minimum-total-energy configuration. The calculated enthalpy of formation is in the $100-\mathrm{meV} /$ atom range for these two superlattices, which is almost an order of magnitude larger than the strain component in $(\mathrm{Si})_{4} /(\mathrm{GaAs})_{2}$. The superlattice dipole induces a metal-insulator transition by periodically tilting the potential. The electrostatic energy derived from this dipole field is the main cause of the instability relative to disproportionation.
\end{abstract}

The growth ${ }^{1-7}$ of GaAs on $\mathrm{Si}(001)$ is technologically significant for high-speed microelectronics and other optical-device applications. In an effort to incorporate the photonics into microelectronics, the growth of a polar semiconductor on a covalent substrate has been achieved. Many applications, such as modulation-doped field-effect transistors, solar cells, single-quantum-well lasers, etc., have already been demonstrated. ${ }^{1}$ The character and the operation of these devices depend on the quality of the interface and there are known problems ${ }^{4}$ with the fabrication of high-quality $\mathrm{Si} / \mathrm{GaAs}$ interfaces. The difference in the electronegativity of the constituents leads to an excess charge at the interface. This gives rise to a substantial dipole field for the polar surfaces. The lattice mismatch between GaAs and $\mathrm{Si}$ also creates misfit dislocations, degrading the quality of the interface even further. Finally, antiphase domain boundaries are created in GaAs due to the existence of monoatomic steps on the $\mathrm{Si}(001)$ surface. These regions contain $\mathrm{Ga}-\mathrm{Ga}$ and $\mathrm{As}-\mathrm{As}$ nonoctet bonds rather than the more favored octet $\mathrm{Ga}-\mathrm{As}$ bonds. The nonoctet bonds act as electrically charged defects. This problem has been solved by heat treatment and a deliberate misorientation of the surface during the growth process. ${ }^{4}$ Antiphase-domain-free GaAs growth on Si substrate has been recently achieved. ${ }^{7}$

The interface of a heterostructure or superlattice between a group-IV elemental ( $\left.A^{\text {IV }}\right)$ and polar III-V compound semiconductor $B^{\mathrm{III}} C^{\mathrm{V}}$ can conveniently be treated in a simple bond-charge picture. ${ }^{4,8}$ The excess charge of the nonoctet $A^{\mathrm{IV}}-C^{\mathrm{V}}$ bond is $Q_{C} \mathrm{v}=-e\left(Z_{C^{\mathrm{v}}}-4\right) / 4$ $\left(Z_{C}\right.$ veing the valence of the anion $\left.C^{\mathrm{v}}\right)$, while the charge of the nonoctet $A^{\text {IV }}-B^{\text {III }}$ bond has been depleted by $A_{B}$ III $=e\left(Z_{B}\right.$ III -4$) / 4$. This leads to a dipole in the supercell which is responsible for the band offset. It also tilts the energy-band diagram along the superlattice direction. The superlattice dipole creates a high electric field, which tends to make the heterostructure unstable. For the same reason, the steps of the odd number of layers on the (001) surface of the substrate give rise to the growth of the domains of polar semiconductors with opposite sublattice allocations. ${ }^{4,5}$ The interface of these domains is called an antiphase boundary. While the excess charge of an $C^{\mathrm{V}}-C^{\mathrm{V}}$ bond at the antiphase boundary is $2 Q_{C} \mathrm{v}$, the charge depletion of a $B^{\mathrm{III}}-B^{\mathrm{III}}$ bond is $2 Q_{B} \mathrm{III}$. The centers with excess charge (or charge depletion) are considered as charged defects at the antiphase boundary. ${ }^{4}$ Moreover, because of the variations in the electronegativity values of the constituent atoms, the cross doping is also expected across the interface. ${ }^{6}$

If the equilibrium lattice constants of the constituent crystals are not significantly different, the lattice misfit can be accommodated by the lattice strain ${ }^{9}$ in the pseudomorphic layers of the grown polar semiconductor. While the grown layers are in registry with the epilayer, the lattice constant in the perpendicular direction expands, leading to a tetragonal distortion. ${ }^{10}$ Owing to the energy barrier associated with the reordering of atoms, pseudomorphic layers can grow prior to the generation of defects. Once the strain energy accumulated by the grown layers exceeds a certain threshold value, the misfit dislocations nucleate. This is another source of defect which affects the quality of the heterostructure.

Here, we consider two systems of particular interest: ${ }^{1}$ namely, $\mathrm{Ge} / \mathrm{GaAs}$ and $\mathrm{Si} / \mathrm{GaAs}$ heterostructures in which the polar semiconductor is restricted to the lateral periodicity of the (001) surface of the elemental (Ge or Si) semiconductor. In the former, the lattice strain is negligibly small because the lattice parameters of $\mathrm{Ge}$ and GaAs are nearly equal. Consequently, the superlattice dipole is the primary source of the instability of the grown GaAs. On the other hand, the lattice constants of Si and GaAs differ by $4 \%$, and thus, in addition to the interface charging, the strain energy is expected to contribute to the instability of the grown layers. ${ }^{11}$ Since the antiphase disorder and the interface charging can be suppressed by the growth on the (211) surface or by step doubling ${ }^{4}$ on 
the $\mathrm{Si}(001)$ surface, the lattice strain with the misfit dislocation generated from it remains to be a severe problem in the pseudomorphic $\mathrm{Si} / \mathrm{GaAs}$ heterostructure.

A number of studies ${ }^{11-19}$ have been carried out for the $\mathrm{Ge} / \mathrm{GaAs}$ interface for the elucidation of the fundamental electronic properties. The change of ionicity across the interface was a key factor whose consequences ${ }^{12,13}$ were explored in some depth. Early on, Harrison et al. ${ }^{11}$ pointed out that a configuration composed of uniform (001) planes of covalent ( $\mathrm{Ge}$ ) and polar ( $\mathrm{Ga}, \mathrm{As}$ ) atoms at the interface is energetically unfavorable and leads to atomic rearrangements at the interface. Based on the empirical-bond-orbital-model ${ }^{8}$ calculations, they proposed an interfacial reconstruction which can reduce the superlattice dipole. To explore the interface structure, Kunc and Martin ${ }^{14}$ studied the compensated interface by using the average-atom approximation. They found that the $\frac{1}{2}(\mathrm{Ge}+\mathrm{As})$ interface is found to be more stable than the $\frac{1}{2}(\mathrm{Ge}+\mathrm{Ga})$ interface, and the band lineup depends strongly on the type of interface.

In the present work, we have investigated some polar interfaces: lattice-matched $\mathrm{Ge} / \mathrm{GaAs}$ and (strained) pseudomorphic $\mathrm{Si} / \mathrm{GaAs}$ superlattices with ideal (uniform) interface, but with optimized interlayer distances. Our objective is to present an analysis of the covalentpolar interface by providing a first-principles value for the superlattice energy. This way, we can evaluate the relative importance of the two factors, i.e., the interface charging (or superlattice dipole) and the strain energy. We have also studied the energy-band structure to understand the origin and confinements of the states near the band edge. Some important findings of our work are as follows: (i) Charge rearrangements occur mainly in the interface region creating a periodic electric field along the superlattice direction; (ii) the contribution of the superlattice dipole to the instability of the $(\mathrm{Si})_{4} /(\mathrm{GaAs})_{2}$ against disproportionation is almost an order of magnitude higher than that of the strain energy; (iii) the valence and conduction bands overlap in momentum space (but not in the direct-lattice space) and pin the Fermi level if the interface is prevented from reconstruction; (iv) the lowest (highest) conduction (valence) -band states are interface states which are derived from $\mathrm{Si}-\mathrm{As}(\mathrm{Si}-\mathrm{Ga}$ ) bonds. These states have significant dispersion if the wave vector $\mathbf{k}$ has a component along $\mathrm{Si}-\mathrm{As}-\mathrm{Si}$ ( $\mathrm{Si}-$ $\mathrm{Ga}-\mathrm{Si}$ ) chains, and hence display a quasi-onedimensional (1D) character.

Our calculations are based on the standard selfconsistent-field (SCF) pseudopotential method, ${ }^{20}$ using nonlocal, norm-conserving pseudopotentials ${ }^{21}$ and Wigner's exchange-correlation potentials. ${ }^{21}$ Bloch states are expanded in terms of plane waves corresponding to a kinetic-energy cutoff $|\mathbf{k}+\mathbf{G}|^{2}=12 \mathrm{Ry}$. SCF calculations are performed by using nine special $\mathbf{k}$ points in the superlattice Brillouin zone (SBZ). Since the local-density approximation predicts a very small band gap for Ge at the chosen kinetic-energy cutoff, we used uniform sampling $(48 \mathrm{k}$ points $)$ in stability analysis of $(\mathrm{Ge})_{4} /(\mathrm{GeAs})_{2}$. For $\mathrm{Si}$ and $\mathrm{Ge}$ substrates, the lattice parameters are determined by the minimization of the bulk total energy with respect to the cubic lattice constants $a$. We found $a_{\mathrm{Si}}^{0} \simeq 10.25$ a.u. (5.42 $\AA$ ), $a_{\mathrm{Ge}}^{0} \simeq 10.66$ a.u. (5.64 $\AA$ ), and $a_{\mathrm{GaAs}}^{0} \simeq 10.66$ a.u. (5.64 $\AA$ ). The pseudomorphic growth of GaAs on the $\mathrm{Si}(001)$ surface is ensured by taking the lateral lattice constant equal to that of the equilibrium $\mathrm{Si}\left(a_{\mathrm{Si}}^{0}\right)$. The lattice constants perpendicular to the epilayer are determined by the minimization of the total energy with respect to the structural degrees of freedom (i.e., Si-Ga, $\mathrm{Si}-\mathrm{As}$, and $\mathrm{Ga}-\mathrm{As}$ interlayer spacings). In this optimization the atomic-force calculations ${ }^{22}$ greatly reduced the computational effort. Since the cubic lattice constants of Ge and GaAs differ only by $\approx 0.01 \%$ and thus the strain energy is negligible, we did not carry out force calculations for this lattice-matched $(\mathrm{Ge})_{4} /(\mathrm{GaAs})_{2}$ superlattice. It is noted that the interface charging may lead to nonuniform bond lengths, perhaps even to buckling, prior to a massive interfacial reconstruction, even if the equilibrium lattice parameters of constituents are lattice matched. ${ }^{19}$

We first determined the volume of the unit cell (or the superlattice vector along the [111] direction, $R_{3}=21.41$ a.u.) of the strained $(\mathrm{Si})_{4} /(\mathrm{GaAs})_{2}$ by scaling the volume of the unit cell of the pseudomorphic $(\mathrm{Si})_{4} /(\mathrm{Ge})_{4}$ superlattice obtained from our earlier optimization. ${ }^{10}$ This is a reasonable approximation because the Poisson ratios of $\mathrm{Ge}$ and GaAs are similar. In the optimization of the atomic configuration of $(\mathrm{Si})_{4} /(\mathrm{GaAs})_{2}$ we then kept the cell volume fixed but varied the interlayer distances until we obtained lowest total energy. The variation of the interlayer spacings in the course of optimization was guided by the atomic (or layer) forces. Our criterion for the optimized structure was satisfied when the magnitudes of the calculated forces are smaller than $\simeq 0.05$ mdyn; this is in conformity with our criterion for the selfconsistency of the charge density. Further optimization of the structure is not meaningful, since the superlattice formation energy per atom changes only $\sim 2 \%$ while the layer forces fluctuate within the noise limits of \pm 0.05 mdyn. The present optimization shows that the interlayer spacings in the strained GaAs sublattice are not uniform and are slightly smaller than what one would obtain from continuum elasticity theory. Earlier, a similar conclusion was obtained for the pseudomorphic $(\mathrm{Si})_{4} /(\mathrm{Ge})_{4}$ superlattice. $^{10}$

The planarly averaged SCF charge density was integrated between the atomic planes along the superlattice direction to obtain the interlayer charge, $Q_{L}$. At the center of the Si sublattice $Q_{L} \simeq 4$ electrons, but it fluctuates in the GaAs sublattice. Interestingly, the charge depletion in the interface between $\mathrm{Si}$ and $\mathrm{Ga}$ layers is $\sim 0.4$ electrons, which is only 0.1 electron smaller than $Q_{\mathrm{Ga}}=e\left(Z_{\mathrm{Ga}}-4\right) / 2$ and leads to a positive charging effect. The excess negative charge in the As/Si interface between $\mathrm{As}$ and $\mathrm{Si}$ layers is $\sim 0.08$ electrons larger than $Q_{\mathrm{As}}=e\left(Z_{\mathrm{As}}-4\right) / 2$. The charge values in these interfaces differ owing to their different interlayer distances.

The energetics and the stability analysis of the superlattice $\left(A^{\mathrm{IV}}\right)_{4} /\left(B^{\mathrm{III}} C^{\mathrm{V}}\right)_{2}$ by comparing ${ }^{10}$ their total energies with those of the constituent crystals. To this end, the total energies of $(\mathrm{Ge})_{4} /(\mathrm{GaAs})_{2}$ and strained $(\mathrm{Si})_{4} /(\mathrm{GaAs})_{2}$ superlattices in their lowest-energy 
configuration (with a uniform interface) are calculated with a strict SCF criterion of $\approx 10^{-6} \mathrm{Ry}$. The total energy calculation of constituents was carried out by using the cubic lattice constants (optimized for the bulk crystals) and tetragonal unit cells similar to that of the superlattices. Therefore, we calculated the total energies of $(\mathrm{Si})_{8},(\mathrm{Ge})_{8},(\mathrm{GaAs})_{4}$, keeping all the other parameters of our calculations (the kinetic-energy cutoff, etc.) the same as in $\left(A^{\mathrm{IV}}\right)_{4} /\left(B^{\mathrm{III}} C^{\mathrm{V}}\right)_{2}$ superlattice. We define the formation energy (or the enthalpy of formation at $T=0 \mathrm{~K}$ ) per atom in the superlattice $\left(A^{\mathrm{IV}}\right)_{4} /\left(B^{\mathrm{III}} C^{\mathrm{V}}\right)_{2}$ as

$$
\begin{aligned}
& \Delta E^{f}\left(\left(A^{\mathrm{IV}}\right)_{4} /\left(B^{\mathrm{III}} C^{\mathrm{V}}\right)_{2}\right)= \frac{1}{8} E_{T}\left(\left(A^{\mathrm{IV}}\right)_{4} /\left(B^{\mathrm{III}} C^{\mathrm{V}}\right)_{2}\right) \\
&-\frac{1}{16}\left[E_{T}\left(\left(A^{\mathrm{IV}}\right)_{8}\right)\right. \\
&\left.+E_{T}\left(\left(B^{\mathrm{III}} C^{\mathrm{IV}}\right)_{4}\right)\right] .
\end{aligned}
$$

Our calculated values for $\Delta E^{f}$ of $(\mathrm{Si})_{4} /(\mathrm{GaAs})_{2}$ and $(\mathrm{Ge})_{4} /(\mathrm{GaAs})_{2}$ are given in Table I. It is seen that the calculated formation energies are among the highest in comparison to many other (001) superlattices ${ }^{10,23-29}$ also listed in Table I. The instability of the heteroepitaxy with uniform atomic planes is therefore clearly suggested. Large values for formation energies have also been obtained by others ${ }^{16,19}$ even for (111) Ge/GaAs superlattices. The formation energy of a typical strained covalent-covalent superlattice [i.e., $(\mathrm{Si})_{4} /(\mathrm{Ge})_{4}$ ] was calculated $^{10}$ to be $\sim 12 \mathrm{meV} /$ atom, out of which $\sim 10 \mathrm{meV}$ arises from the strain energy of the Ge sublattice undergoing a tetragonal distortion. The formation energy of the $(\mathrm{Si})_{4} /(\mathrm{GaAs})_{2}$ is seen to be several times larger than that of $(\mathrm{Si})_{4} /(\mathrm{Ge})_{4}$.

The strain-energy contribution in the total formation energy of the GaAs pseudomorphically grown on $\mathrm{Si}(001)$ is small. This can be seen from an approximate value for the contribution of the strain energy which can be deduced from the equation

$$
\Delta E^{s}=\frac{1}{8}\left[E_{T}^{s}\left((\mathrm{GaAs})_{4}\right)-E_{T}^{0}\left((\mathrm{GaAs})_{4}\right)\right],
$$

where $E_{T}^{s}\left((\mathrm{GaAs})_{4}\right)$ is calculated for GaAs crystal laterally restricted to the lattice parameters of $\mathrm{Si}$, but with a tetragonal distortion as in $(\mathrm{Si})_{4} /(\mathrm{GaAs})_{2}$. The calculated value of the strain energy $\Delta E^{s}$ of about $13 \mathrm{meV}$ per atom is indeed much smaller than $\Delta E^{f}$. Note that the

TABLE I. Formation enthalpy (meV/atom) for various (001) superlattices.

\begin{tabular}{lll}
\hline \multicolumn{1}{c}{ Superlattice } & \multicolumn{1}{c}{$\Delta E^{f}$} & Reference \\
\hline$(\mathrm{GaAs})_{1} /(\mathrm{AlAs})_{1}$ & $2.3,2.7,8.8$ & $23,24,25$ \\
$(\mathrm{HgTe})_{1} /(\mathrm{CdTe})_{1}$ & 3.0 & 26 \\
$(\mathrm{GaP})_{1} /(\mathrm{InP})_{1}$ & $-6.3,13.6,22.8,28.9$ & $27,28,29,24$ \\
$(\mathrm{GaAs})_{1} /(\mathrm{InAs})_{1}$ & 20.9 & 24 \\
$(\mathrm{GaAs})_{1} /(\mathrm{GaSb})_{1}$ & 32.3 & 24 \\
$(\mathrm{Si})_{4} /(\mathrm{Ge})_{4}$ & 11.9 & 30 \\
$(\mathrm{GaAs})_{5} /(\mathrm{Ge})_{2}$ & 40.0 & 17 \\
$(\mathrm{GaAs})_{2} /(\mathrm{Si})_{4}$ & 92.5 & 19 \\
$(\mathrm{GaAs})_{2} /(\mathrm{Ge})_{4}$ & 65.0 & 19 \\
$(\mathrm{GaAs})_{2} /(\mathrm{Si})_{4}$ & 86.9 & Present \\
$(\mathrm{GaAs})_{2} /(\mathrm{Ge})_{4}$ & 67.1 & Present \\
\hline \hline
\end{tabular}

strain energy estimated from elasticity theory is also small (i.e., only $\sim 5 \mathrm{meV} /$ atom). What emerges from Table $I$ is that whenever different cation (anion) in a common anion (cation) superlattice belongs to the same column of the Periodic Table the formation enthalpy is dominated by the lattice mismatch. For compound superlattices formed with elements from different columns of the Periodic Table, the instability is dominated by the superlattice dipole. The strain-energy contribution is usually masked by the dipole contribution. Interestingly enough, most superlattices are either unstable or at best metastable. Clearly, large values for the formation energies arise from the electrostatic energy due to the superlattice dipoles ${ }^{11,16-19}$ and are the major cause of the instability of the heteroepitaxy.

In the pseudomorphic $(\mathrm{Si})_{n} /(\mathrm{Ge})_{n}$ superlattices, the formation energy was found ${ }^{9,10}$ to increase with increasing Ge-sublattice thickness. This is so because Si and Ge are isovalent and the lattice strain is the principal component of the formation energy. The misfit dislocations were found ${ }^{10}$ to form for $n>6$ in $(\mathrm{Si})_{n} /(\mathrm{Ge})_{n}$ superlattice. In $(\mathrm{Si})_{4} /(\mathrm{GaAs})_{2}, \Delta E^{f}$ is about six times larger than the threshold energy. One must then expect significant rearrangements of atoms at the interface to reduce the dipole field.

We also investigated the Ge layer between $\mathrm{Ga}$ and As layers in the GaAs sublattice. To this end we compared the calculated total energies of GeGaGeAs and $(\mathrm{GaAs})_{1} /(\mathrm{Ge})_{2}$ superlattices, and found that the intervening $\mathrm{Ge}$ layer between $\mathrm{Ga}$ and As layers in GaAs is energetically highly unfavorable. This result is in agreement with the calculations by Lee, Bylander, and Kleinman. ${ }^{18}$ In the case of $\mathrm{GeGaGeAs}$ the formation energy increases due to the dipole field induced by the nonoctet $\mathrm{Ge}-\mathrm{Ga}$ and $\mathrm{Ge}-\mathrm{As}$ bonds replacing the octet $\mathrm{Ga}-\mathrm{As}$ bonds in the GaAs sublattice. In contrast to that, the interface

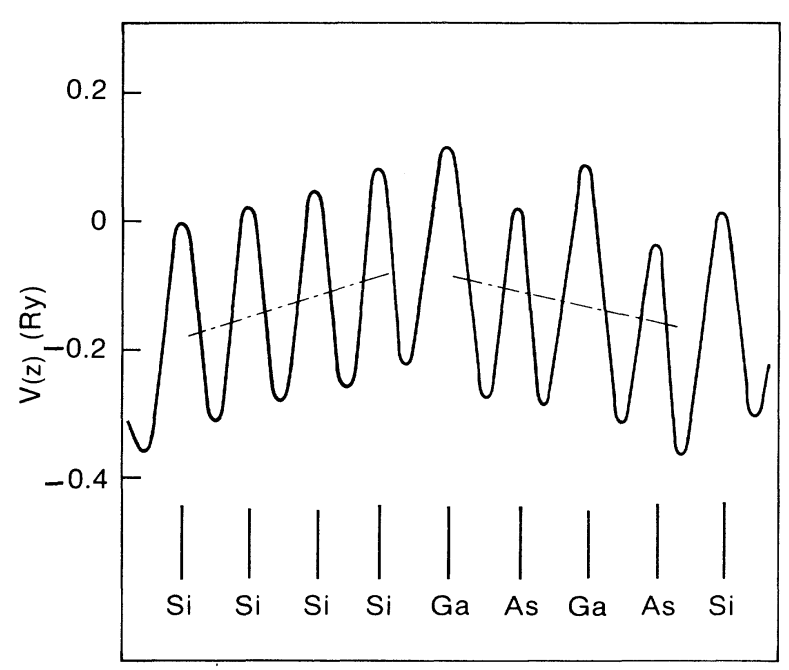

FIG. 1. Planarly averaged SCF pseudopotential $V(z)$ for $(\mathrm{Si})_{4} /(\mathrm{GaAs})_{2}$. An estimate of the mean value is shown by dashed-dotted lines. 

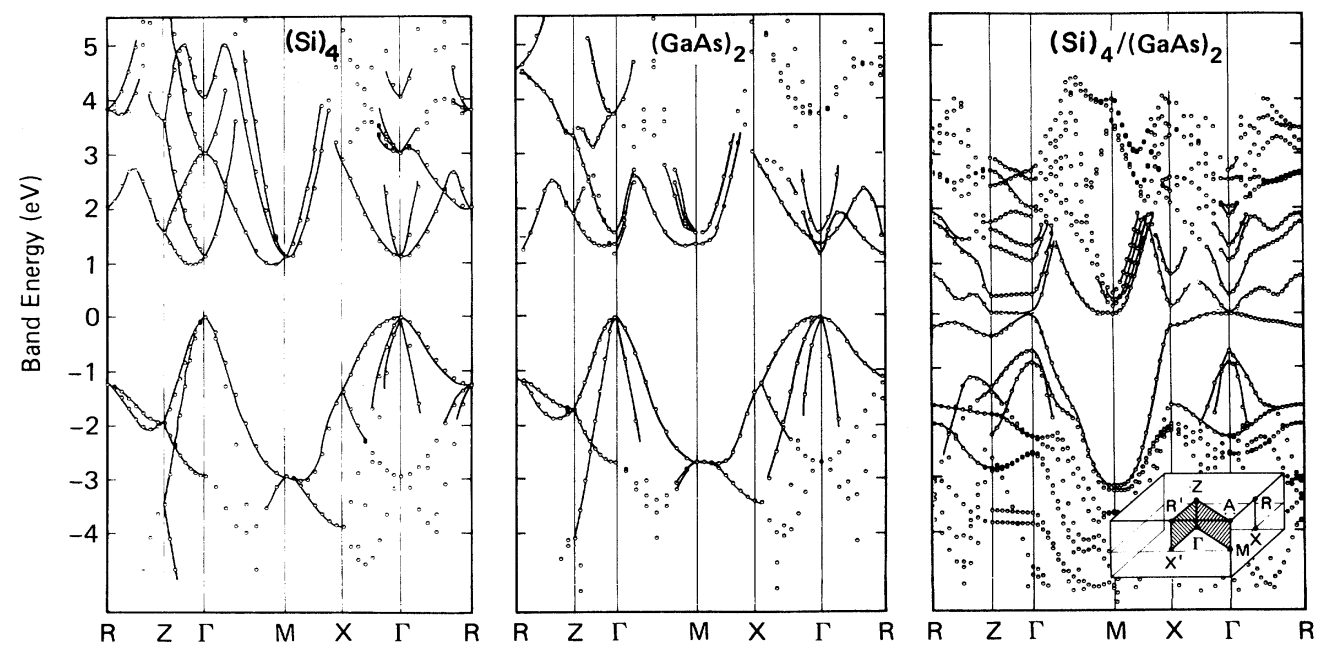

FIG. 2. Energy band structures (a) $(\mathrm{Si})_{4},\left(\right.$ b) $(\mathrm{GaAs})_{2}$, (c) $(\mathrm{Si})_{4} /(\mathrm{GaAs})_{2}$. The inset shows the superlattice Brillouin zone (SBZ) corresponding to the tetragonal unit cell. The zero of energy is taken at the maximum of the valence bands.

charging and the dipole field can be reduced and hence the formation energy ${ }^{11}$ is lowered as a result of welldefined rearrangement of $\mathrm{Ge}, \mathrm{Ga}$, and $\mathrm{As}$ atoms at the interface leading to the reconstruction of the interface.

In Fig. 1 we present the variation of the $1 \mathrm{D}$ potential energy $V(z)$ along the superlattice axis of $(\mathrm{Si})_{4} /(\mathrm{GaAs})_{2}$. This potential-energy curve is obtained by planarly averaging the SCF pseudopotential. Owing to interface charging, the mean value of $V(z)$ displays a sawtooth form with a significant tilt. Starting from the lowest value at the $\mathrm{As} / \mathrm{Si}$ interface, it rises towards the highest value in the $\mathrm{Si} / \mathrm{Ga}$ interface, and thereafter it is lowered by going to the As/Si interface. We used a simple model to explain this behavior. We represented $(\mathrm{Si})_{4} /(\mathrm{GaAs})_{2}$ as a continuous media composed of two types of dielectric slabs with $+0.4 e$ and $-0.4 e$ charges uniformly distributed in the space equivalent to the $\mathrm{Si} / \mathrm{Ga}$ and $\mathrm{As} / \mathrm{Si}$ interfaces, respectively. The resulting voltage drop across the dielectric slabs was calculated to be $0.9 \mathrm{eV}$, which is in good agreement with the SCF calculations. Following the macroscopic averaging scheme $\mathrm{e}^{31}$ it is easy to see that one could get band offsets in the range of $\sim 1 \mathrm{eV}$. One also gets important electronic effects as outlined below.

In Fig. 2 we present the band structure of $\left(\mathrm{Si}_{4}\right.$, $(\mathrm{GaAs})_{2}$, and $\left(\mathrm{Si}_{4} /(\mathrm{GaAs})_{2}\right.$ all calculated in the tetragonal cell. Owing to the superstructure, the lowest conduction band of $\mathrm{Si}$ for $\mathbf{k} \|[001]$ has experienced folding along the $\Gamma Z$ direction of the SBZ. As a result, the conductionband minima occur not only along the $\Gamma M$ direction but also along the $\Gamma Z$ direction. Bands of $(\mathrm{GaAs})_{2}$ experience similar foldings. Upon the superlattice formation, the bands of $(\mathrm{GaAs})_{2}$ in $(\mathrm{Si})_{4} /(\mathrm{GaAs})_{2}$ undergo changes and splittings due to the tetragonal strain. Moreover, because of the natural band lineup and the interface dipole, the bands of $(\mathrm{GaAs})_{2}$ are shifted relative to the bands of $(\mathrm{Si})_{4}$. The electric field induced by the interface charge gives rise to dramatic tilting in the band diagram in the real

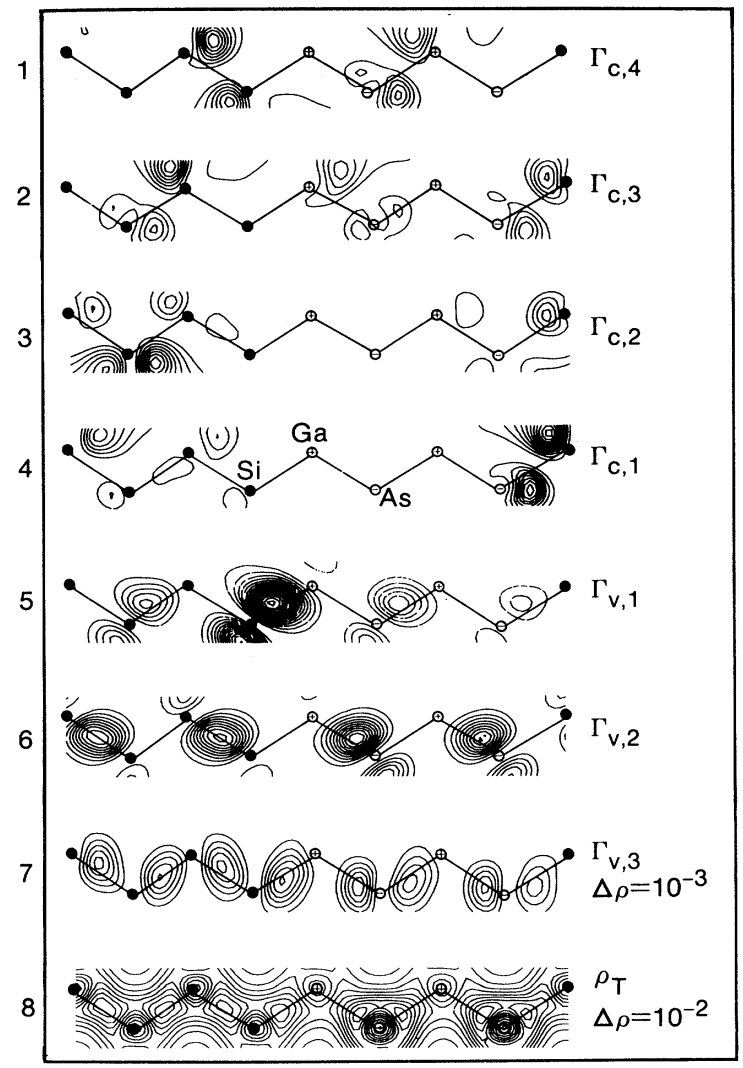

FIG. 3. Contour plots of the SCF charge density calculated for $(\mathrm{Si})_{4} /(\mathrm{GaAs})_{2}$. Total charge density $\rho_{T}$ and state charge densities of the valence- and conduction-band states at the $\Gamma$ point. $\Gamma_{v, 1}$ and $\Gamma_{c, 1}$ are the highest valence-band and the lowest conduction-band states, respectively. Contour spacings $\Delta \rho$ in electrons $/(\text { a.u. })^{3}$ are indicated. 
space. In Fig. 2(c) we observe a negative band gap in momentum space due to superlattice formation. The same bands at the edge of the conduction band are flat along the superlattice direction but they have a parabolic dispersion for $\mathbf{k}$ lying in a (001) plane. This is a characteristic feature of a $2 \mathrm{D}$ electron system.

It is appropriate to make some general comments on the electronic structure of these heterostructures. (i) It is well known that the band gaps are underestimated by our calculations which use the local-density approximation. The difference between the experiment and calculations for $\mathrm{Si}$ and $\mathrm{Ge}$ is $\sim 0.5 \mathrm{eV}$, which is usually compensated by applying a constant upwards shift to the conductionband energies. In the present case, the conduction and valence bands of $\left(\mathrm{Si}_{2 m} /(\mathrm{GaAs})_{m}\right.$ may not overlap for $m=2$. They would certainly overlap for $m=3$, since the strength of the dipole increases with $m$. The bands shown in Fig. 2 have therefore not been shifted. (ii) Owing to the overlap of the valence band with the conduction band, the system undergoes a metal-insulator transition. The overlap occurs only in the momentum space; these bands are separated in the real space, however. Then conduction along the superlattice direction occurs via tunneling. (iii) If the ideal $\left(A^{\mathrm{IV}}\right)_{2 m} /\left(B^{\mathrm{III}} C^{\mathrm{V}}\right)_{m}$ superlattice could somehow be stabilized one would observe interesting transport properties. For example, the metallic state would undergo a further metal-insulator transition ${ }^{32}$ opening a small gap. The excitons created in this superlattice would display behavior similar to that recently observed, ${ }^{33}$ in which the photoluminescence linewidth is suddenly reduced below a critical temperature.

The origin and the localization of the states of $(\mathrm{Si})_{4} /(\mathrm{GaAs})_{2}$ are examined by the charge densities presented in Fig. 3. Significant changes of the charge density at the interface are depicted in the contour plots of the total charge density. The topmost valence-band state is localized near the interface region on the $\mathrm{Si}-$ $\mathrm{Ga}-\mathrm{Si}$ bonds. Therefore, the band has comparatively low dispersion for $\mathbf{k}$ perpendicular to the bond plane (i.e., $X \Gamma$ direction). This is an interface band which is split off from the valence-band continua and is localized in the hole quantum well consisting of bowed $\mathrm{Si}$ and GaAs valence-band edges. Because of size effects, the second and third valence-band states do not display any confined character. The lowest conduction band is derived from As and $\mathrm{Si}$, and thus is localized in the interface region. This band has a minimum at the $\Gamma$ point, and is almost flat along the $\Gamma Z$ direction. This state is confined in the lowest corner of the quantum well made by the tilting of $\mathrm{Si}$ and GaAs conduction-band edges. The second and third conduction-band states are primarily confined in the $\mathrm{Si}$ sublattice and have flat bands along $\Gamma-Z$, and a (parabolic) subband structure in the plane $\mathbf{k} \|[001]$ and around the $\Gamma$ point.

In conclusion, the results of the SCF total-energy calculations indicate that the superlattice dipole and the electric field induced from it play a crucial role in stability (or lack of it), as well as in the electronic structure of the covalent-polar semiconductor superlattices. The strain energy arising from the lattice mismatch is found to be a less significant contribution as far as the stability is concerned. None of the superlattices are found to be stable against disproportionation. The dipoles tend to destroy themselves ${ }^{11}$ to some extent by inducing a lattice rearrangement or negative band gap.
*Electronic address: IPBATRA @ ALMVMD.

†Present address: Department of Electrical Engineering, Stanford University, Stanford, CA 94305.

${ }^{1}$ The current status of the field is reviewed in Heterostructures on Silicon: One Step Further with Silicon, Vol. 160 of NATO Advanced Study Institute, Series E: Applied Sciences, edited by Y. I. Nissim and E. Rosencher (Plenum, New York, 1989).

${ }^{2}$ See, for example, S. M. Koch, S. J. Rosner, R. Hull, G. W. Yoffe, and J. S. Harris, Jr., J. Cryst. Growth 81, 205 (1987); B. Bourguignon, K. L. Carleton, and S. R. Leone, Surf. Sci. 204, 455 (1988); K. Adomi, S. Strite, and H. Morkoc, Appl. Phys. Lett. 56, 469 (1990).

${ }^{3}$ L. Tapfer and K. Ploog, Phys. Rev. B 33, 5565 (1986).

${ }^{4}$ H. Kroemer, J. Cryst. Growth 81, 193 (1987).

${ }^{5}$ P. R. Pukite and P. I. Cohen, J. Cryst. Growth 81, 214 (1987).

${ }^{6}$ A. T. Fiory, J. C. Bean, L. C. Feldman, and I. K. Robinson, J. Appl. Phys. 56, 1227 (1984).

${ }^{7}$ M. Tachikawa and M. Yaguchi, Appl. Phys. Lett. 56, 484 (1990).

${ }^{8}$ W. A. Harrison and S. Ciraci, Phys. Rev. B 10, 1516 (1974); Electronic Structure and the Properties of Solids, edited by W. A. Harrison (Freeman, San Francisco, 1980).

${ }^{9}$ C. H. Van de Walle and R. M. Martin, J. Vac. Sci. Technol. B 3, 1256 (1985); Phys. Rev. B 34, 5621 (1986); 37, 4108 (1987).

${ }^{10}$ S. Ciraci and I. P. Batra, Phys. Rev. B 38, 1835 (1988); S. Cira- ci, I. P. Batra, and E. Tekman, ibid. 38, 12728 (1988); S. Ciraci, A. Baratoff, and I. P. Batra, ibid. 41, 6069 (1990). See also Band Structure Engineering in Semiconductor Microstructures, Vol. 189 of NATO Advanced Study Institute, Series B: Physics, edited by R. A. Abram and M. Jaros (Plenum, New York, 1989).

${ }^{11}$ W. A. Harrison, E. A. Kraut, J. R. Waldrop, and R. W. Grant, Phys. Rev. B 18, 4402 (1978).

${ }^{12}$ G. A. Baraff, J. A. Appelbaum, and D. R. Hamann, Phys. Rev. Lett. 38, 237 (1977); J. Vac. Sci. Technol. 14, 999 (1977).

${ }^{13}$ W. E. Pickett, S. G. Louie, and M. L. Cohen, Phys. Rev. B 17, 815 (1978); J. Ihm and M. L. Cohen, ibid. 20, 729 (1979).

${ }^{14}$ K. Kunc and R. Martin, Phys. Rev. B 24, 3445 (1981).

${ }^{15}$ J. C. Duran, A. Muñoz, and F. Flores, Phys. Rev. B 35, 7721 (1987); N. E. Christensen, ibid. 37, 4528 (1988).

${ }^{16} \mathrm{~S}$. Baroni, R. Resta, and A. Baldereschi, Proceedings of the 19th International Conference on the Physics of Semiconductors, Warsaw, Poland (1988) (to be published).

${ }^{17}$ A. Muñoz, N. Chetty, and R. M. Martin, Phys. Rev. B 41, 2976 (1990).

${ }^{18}$ D. M. Bylander and L. Kleinman, Phys. Rev. B 40, 3509 (1990); S. Lee, D. M. Bylander, and L. Kleinman, ibid. 41, 10264 (1990).

${ }^{19}$ R. G. Dandrea, S. Froyen, and A. Zunger, Phys. Rev. B 42, 3213 (1990). 
${ }^{20}$ J. Ihm, A. Zunger, and M. L. Cohen, J. Phys. C 12, 4409 (1979).

${ }^{21}$ G. B. Bachelet, D. R. Hamann, and M. Schlüter, Phys. Rev. B 26, 4199 (1982). These nonlocal pseudopotentials were obtained by using Ceperley-Alder exchange-correlation potential [see D. M. Ceperley and and B. J. Alder, Phys. Rev. Lett. 45, 566 (1980)], but underestimates the lattice parameters for $\mathrm{Ge}$ and GaAs. By using Wigner's exchange-correlation potential, we were able to obtain good values for lattice parameters and thus to avoid artifical strain energy in the calculations. Since we are calculating difference energies and dealing with large electrostatic energies, Wigner's exchangecorrelation potential does not affect our conclusions in any essential manner.

${ }^{22}$ I. P. Batra, S. Ciraci, G. P. Sirivastava, J. S. Nelson, and C. Y. Fong, Phys. Rev. B 34, 8246 (1986)

${ }^{23}$ D. M. Bylander and L. Kleinman, Phys. Rev. B 34, 5280 (1986).

${ }^{24}$ P. Boguslawski and A. Baldereschi, Proceedings of the 19th International Conference on the Physics of Semiconductors, Warsaw, Poland, 1988 (Ref. 16).

${ }^{25}$ S. Ciraci and I. P. Batra, Phys. Rev. Lett. 58, 2114 (1987); Phys. Rev. B 36, 1225 (1987); I. P. Batra, S. Ciraci, and J. S.
Nelson, J. Vac. Sci. Technol. B 5, 1300 (1987).

${ }^{26}$ D. M. Wood, S.-H. Wei, and A. Zunger, Phys. Rev. Lett. 58, 1123 (1987).

${ }^{27}$ A. A. Mbaye, L. G. Ferreira, and A. Zunger, Phys. Rev. Lett. 58, 49 (1987); G. P. Srivastava, J. L. Martins, and A. Zunger, Phys. Rev. B 31, 2561 (1985).

${ }^{28}$ J. S. Nelson and I. P. Batra, Phys. Rev. B 39, 3250 (1989).

${ }^{29}$ J. E. Bernard, S.-H. Wei, D. M. Wood, and A. Zunger, Bull. Am. Phys. Soc. 33, 481 (1988).

${ }^{30}$ D. W. Niles, G. Margaritondo, P. Perfetti, C. Quaresima, and M. Capozzi, Appl. Phys. Lett. 47, 1092 (1985).

${ }^{31}$ A. Baldereschi, S. Baroni, and R. Resta, Phys. Rev. Lett. 61, 734 (1988). In the present study the sublattice $(\mathrm{GaAs})_{2}$ is not thick enough to calculate the value of the band offset accurately.

${ }^{32}$ B. I. Halperin and T. M. Rice, in Solid State Physics: Advances in Research and Applications, edited by $\mathrm{H}$. Ehrenreich, F. Seitz, and D. Turnbull (Academic, New York, 1968), Vol. 21, p. 115.

${ }^{33}$ T. Fukuzawa, E. E. Mendez, and J. M. Hong, Phys. Rev. Lett. 64, 3066 (1990); see also Z. Gedik and S. Ciraci, J. Phys. Condens. Matter 2, 8985 (1990). 